\title{
Nip New Disease Reports \\ First report of stem canker of dragon fruit caused by Alternaria spp. in Taiwan
}

\author{
X.L. Jin ${ }^{1 *}$, Y.Z. Ko ${ }^{1}$, M.S. Siti Nordahliawate ${ }^{2}$, M.H. Mohd ${ }^{3}$ and Y.C. Chiang ${ }^{1,4}$
}

${ }^{1}$ Department of Biological Sciences, National Sun Yat-Sen University, Lienhai Road, Kaohsiung, 80424, Taiwan ; ${ }^{2}$ Laboratory for Pest, Disease and Microbial Biotechnology (LAPDiM), School of Food Science and Technology, Universiti Malaysia Terengganu, Kuala Terengganu, 21030, Malaysia ; ${ }^{3}$ School of Biological Sciences, Universiti Sains Malaysia, Penang, 11800, Malaysia ; ${ }^{4}$ Department of Biomedical Science and Environment Biology, Kaohsiung Medical University, Kaohsiung 807, Taiwan

*E-mail: shelleyjin25@gmail.com

Received: 16 Apr 2020. Published: 19 Jun 2020. Keywords: fungal plant disease, Hylocereus polyrhizus

Red-fleshed dragon fruit (Hylocereus polyrhizus) is an important agriculture commodity in Taiwan, and it is affected by diseases such as stem canker and stem rot (Mohd et al., 2014). In September, round, orange to reddish lesions with dark red to black centres were observed on $70 \%$ of dragon fruit stems on two farms in Pingtung, southern Taiwan. The lesions merged and formed larger diseased areas on the stem surface (Fig. 1). The disease can cause significant loss of fruit when subsequently kept in cold storage (commercially at $8_{-}^{\circ} \mathrm{C}$ ) (Fig. 2).

Small pieces $\left(3-5 \mathrm{~mm}^{2}\right)$ of diseased tissues were cut from the stems and surface-sterilised with a $75 \%$ ethanol swab, soaked in $1 \%$ sodium hypochlorite $(\mathrm{NaOCl})$ for $2 \mathrm{~min}$, and rinsed in several changes of sterile distilled water for $1 \mathrm{~min}$ each. Subsequently, the surface-sterilised tissues were placed onto half strength potato dextrose agar (PDA) and incubated at room temperature $\left(25^{\circ} \mathrm{C}\right)$ with alternating 12-hr daylight and 12-hr dark. After 5 to 7 days, two distinct greyish and brownish colonies grew on PDA (Fig. 3). Conidia of both colonies were light-brown, obclavate, 15.0 to 55.0 $\pm 5.4 \mu \mathrm{m}$ long and 5.5 to $12.2 \pm 1.6 \mu \mathrm{m}(n=50)$ wide. There were 2 to 6 transverse septa on the conidia (Fig. 4). Based on these morphological characteristics, the isolates were identified as Alternaria spp. (Simmons, 2007).

Genomic DNA of 11 isolates [four greyish colonies (WHHY) and seven brownish colonies (BLHY)] were extracted from pure cultures using a Genomic DNA Extraction Kit (RBC Bioscience Corp., Taiwan). Primers ITS1/4 (512 bp), Alt-for/rev (471 bp), and gpd1/2 (432 bp) were used in PCR to amplify the internal transcribed spacer (ITS) region, and major allergen Alt a 1 (Alt), and glyceraldehyde-3-phosphate dehydrogenase (GAPDH) genes, respectively. Amplicons of the expected size were amplified from all isolates and were subsequently sequenced (GenBank Accession Nos. MK874994-MK875004; MK881612-MK881622; MT135547-MT135557). The sequences of the WHHY and BLHY isolates had 99-100\% identity to A. burnsii (MH865506.1, ITS); KP123967.1, Alt); JQ646305.1. GAPDH)) and 99-100\% identity to A. tomato (KP124446.1, IT); KP123991, Alt; KP124294.1, GAPDH), respectively. Reconstruction of the neighbour-joining and maximum likelihood phylogenetic tree was based on the combined dataset and reference CBS strains (Fig. 5). WHHY

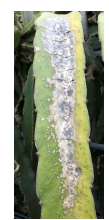

Figure 1

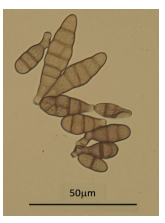

and BLHY isolates clustered most closely with A. burnsii and A. tomato but showed significant differences in phylogenetic lineages from the other Alternaria spp., thus, both greyish and brownish isolates could be new species.

Pathogenicity tests were done by wounding the stem using a sterilised needle on ten healthy detached stems of dragon fruit. The conidial inoculum $\left(1 \times 10^{5}\right.$ conidia/ml, taken from 14 -day-old cultures $)$ was dropped onto the wounded stems. Controls were treated with sterile distilled water. Inoculated stems and controls were kept in a damp chamber to maintain high humidity $(97 \%)$ at room temperature $\left(25^{\circ} \mathrm{C}\right)$. All stems were sprayed with sterile distilled water every day. After three to four days, tiny red spots appeared on the inoculated stems and these spots enlarged 20 to 60 days after inoculation, resembling those observed on dragon fruit stems in the field. No symptoms were observed on the control stems. The symptoms on stems inoculated with the greyish and brownish isolates could not be differentiated.The pathogenicity test was repeated three times with the same results. To our knowledge, this is the first report of Alternaria spp. on Hylocereus polyrhizus in Taiwan.

\section{Acknowledgements}

This research was supported by funding from the Ministry of Science and Technology, Taiwan, MOST 105-2621-B-110-003-MY3 and MOST 107-2313-B-110-004 to YCC, grants from Ministry of Education, Taiwan, and partial grants from NSYSU Higher Education Sprout Project.

\section{References}

1. Mohd, M. H., Salleh, B. \&, \& Zakaria, L. (2014). An overview of fungal diseases of pitaya in malaysia. Improving Pitaya Production and Marketing, 87 -93. https://www.fftc.org.tw/htmlarea_file/activit ies/20150817121105/09-15P10.pdf. Accessed 15 April 2020.

2. Simmons EG, 2007. Alternaria: An Identification Manual. Utrecht, Netherlands: CBS Fungal Biodiversity Centre.

Figure 4

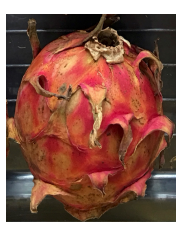

Figure 2

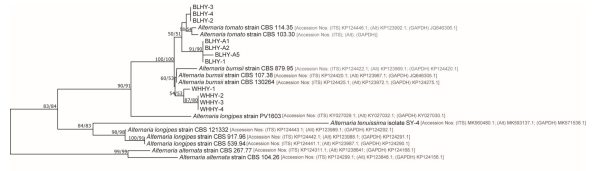

Figure 5

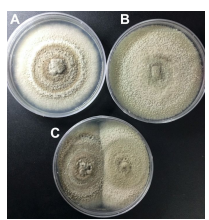

Figure 3

To cite this report: Jin XL, Ko YZ, Siti Nordahliawate MS, Mohd MH, Chiang YC, 2020. First report of stem canker of dragon fruit caused by Alternaria spp. in Taiwan. New Disease Reports 41, 35. http://dx.doi.org/10.5197/j.2044-0588.2020.041.035 